\title{
Distance rationalizability of scoring rules
}

\author{
Citation for published version (APA):
}

Can, B. (2013). Distance rationalizability of scoring rules. Maastricht University, Graduate School of Business and Economics. GSBE Research Memoranda No. 028 https://doi.org/10.26481/umagsb.2013028

Document status and date:

Published: 01/01/2013

DOI:

10.26481/umagsb.2013028

Document Version:

Publisher's PDF, also known as Version of record

\section{Please check the document version of this publication:}

- A submitted manuscript is the version of the article upon submission and before peer-review. There can be important differences between the submitted version and the official published version of record.

People interested in the research are advised to contact the author for the final version of the publication, or visit the DOI to the publisher's website.

- The final author version and the galley proof are versions of the publication after peer review.

- The final published version features the final layout of the paper including the volume, issue and page numbers.

Link to publication

\footnotetext{
General rights rights.

- You may freely distribute the URL identifying the publication in the public portal. please follow below link for the End User Agreement:

www.umlib.nl/taverne-license

Take down policy

If you believe that this document breaches copyright please contact us at:

repository@maastrichtuniversity.nl

providing details and we will investigate your claim.
}

Copyright and moral rights for the publications made accessible in the public portal are retained by the authors and/or other copyright owners and it is a condition of accessing publications that users recognise and abide by the legal requirements associated with these

- Users may download and print one copy of any publication from the public portal for the purpose of private study or research.

- You may not further distribute the material or use it for any profit-making activity or commercial gain

If the publication is distributed under the terms of Article $25 \mathrm{fa}$ of the Dutch Copyright Act, indicated by the "Taverne" license above, 


\section{Maastricht University}

Burak Can

Distance rationalizability of scoring rules

RM/ 13/028

\section{GSBE}

Maastricht University School of Business and Economics

Graduate School of Business and Economics

P.O Box 616

NL- 6200 MD Maastricht

The Netherlands 


\title{
Distance Rationalizability of Scoring Rules
}

\author{
Burak Can*
}

November 2012

\begin{abstract}
Collective decision making problems can be seen as finding an outcome that is "closest" to a concept of "consensus". Nitzan (1981) introduced "Closeness to Unanimity Procedure" as a first example to this approach and showed that the Borda rule is the closest to unanimity under swap distance (a.k.a the Kemeny (1959) distance). Meskanen and Nurmi (2008) shows that the Dodgson rule is the closest to Condorcet under swap distance. Elkind et al. (2009, 2012) generalized this concept as distance-rationalizability, where being close is measured via various distance functions and with many concepts of consensus, e.g., unanimity, Condorcet etc. In this paper, we show that all non-degenerate scoring rules can be distance-rationalized as "Closeness to Unanimity" procedures under a class of weighted distance functions introduced in Can (2012). Therefore, the results herein generalizes, partly, the results in Nitzan (1981) and complements the extensive findings in Elkind et al. (2009).
\end{abstract}

JEL classification:

Keywords: Strict preferences; Rankings; Distance-rationalizability

\section{Introduction}

Nitzan (1981) introduced the closeness to unanimity procedures (CUPs) for collective decision making problems. Given a distance function - for the concept of closeness - over preference profiles, these procedures finds "closest" unanimous preference profiles to the original preference profile at hand. This approach, in a sense, yields the outcome which requires the minimal total compromise towards a unanimous agreement from a utilitarian perspective.

Meskanen and Nurmi (2008) uses other consensus concepts such as the existence of Condorcet winner in a profile. Then the compromise needed is not to achieve a unanimous profile but to achieve a Condorcet winner as least costly as possible. They show that if the consensus concept is not unanimity but only a Condorcet winner, then the Dodgson winner in a profile is the closest to the Condorcet winner under a compromise defined as the swap distance.

Elkind et al. $(2009,2012)$ generalize the notion of closeness to various concepts of consensus as distance-rationalization. They use many reasonable consensus classes apart from unanimity, and employ different distance functions to shed light on the existing collective choice rules and their relation to distance functions within a consensus approach.

${ }^{*}$ Department of Economics, School of Business and Economics, Maastricht University, P.O. Box 616, 6200 MD Maastricht, The Netherlands; E-mail: b.can@maastrichtuniversity.nl, Phone number: +31 433883807 . I would like to thank the Netherlands Organisation for Scientific Research (NWO) for the grant with project nr. 400-09-354. 
In this paper, we focus on one particular class of rules, the scoring rules. Nitzan (1981) showed that the simplest scoring rule, i.e. the Borda rule, is equivalent to closeness to unanimity procedure under swap distance. Elkind et al. (2009) extend this result, among other rules, to all scoring rules, however, under pseudo-distances 1 . We show that the class of non-degenerate ${ }^{2}$ scoring rules can in fact be distance-rationalized via a class of functions introduced in Can (2012) which satisfy also (iii) the symmetry condition and therefore are regular distance functions. This complements the extensive survey in Elkind et al. (2009) over distance-rationalization of many well-known rules, in particular the scoring rules.

\section{Model}

\subsection{Preliminaries}

Let $N$ be a finite set of agents with cardinality $n$, and $A$ be the set of finite set of alternatives with cardinality $m$. The set of all possible strict preferences, i.e., complete, transitive and antisymmetric binary relations over $A$, is denoted by $\mathcal{L}$. A generic preference is denoted by $R \in \mathcal{L}$ whereas the set of strict preferences with an alternative $a$ at the top is denoted by $\mathcal{L}^{a}$. A preference profile is an $n$-tuple vector of preferences denoted by $p=(p(1), p(2), \ldots, p(n)) \in \mathcal{L}^{N}$. Given an alternative $a \in A$, we denote profiles with $a$ as the top alternative in each individual preference by $p^{a}$.

For $l=1,2, \ldots, m, R(l)$ denotes the alternative in the $l^{\text {th }}$ position in $R$, e.g., $R(1)$ denotes the top alternative. Given an alternative $a$ and a preference $R$, we denote the position of $a$ in $R$ by $a(R)$, i.e., $a(R)=x$ if and only if $x=R(a)$. To denote the position of alternative $a$ in the preference of $i^{\text {th }}$ individual in a profile, we abuse notation and write $a(i)$ instead of $a(p(i))$, as long as it is clear which preference profile we refer to. Two linear orders $\left(R, R^{\prime}\right) \in \mathcal{L}^{2}$ form an elementary chang $\oint^{3}$ in position $k$ whenever $R(k)=R^{\prime}(k+1), R^{\prime}(k)=R(k+1)$ and for all $t \notin\{k, k+1\}$, $R(t)=R^{\prime}(t)$, i.e. $\left|R \backslash R^{\prime}\right|=1$. Given any two distinct linear orders $R, R^{\prime} \in \mathcal{L}$, a vector of linear orders $\rho=\left(R_{0}, R_{1}, \ldots, R_{k}\right)$ is called a path between $R$ and $R^{\prime}$ if $k=\left|R \backslash R^{\prime}\right|, R_{0}=R, R_{k}=R^{\prime}$ and for all $i=1,2, \ldots k,\left(R_{i-1}, R_{i}\right)$ forms an elementary change. For the special case where $R=R^{\prime}$, we denote the unique path as $\rho=(R, R)$.

A vector $s=\left(s_{1}, s_{2}, \ldots, s_{m}\right)$ over positions of alternatives in a preferences is called a scoring vector whenever $s_{1} \geq s_{2} \geq \ldots s_{m} \geq 0$. A scoring vector $s$ is called non-degenerate if scores are strictly decreasing from $s_{1}$ to $s_{m}$, i.e., $s_{1}>s_{2}>\ldots s_{m} \geq 0$. The score of an alternative $a$ in a preference $R$ is denoted by $\operatorname{score}(a, R)$ and is equal to $s_{a(R)}$ in the scoring vector.

A collective choice rule, or a voting rule, is a correspondence $\alpha: \mathcal{L}^{N} \rightarrow 2^{A} \backslash \emptyset$, which assigns each preference profile a nonempty subset of alternatives. Given a preference profile $p \in \mathcal{L}^{N}$, a scoring rule, denoted by $\alpha_{s}$, with scoring vector $s$ is a choice rule that assigns a summed score to each alternative in $A$ as: $\sum_{i \in N} \operatorname{score}(a, p(i))$ and assigns to each profile the alternatives with

\footnotetext{
${ }^{1}$ A distance function (also called a metric) satisfies (i) non-negativity, (ii) identity of indiscernibles, (iii) symmetry, and (iv) the triangular inequality. A pseudo-distance function satisfies all but the third, i.e., (iii) the symmetry condition.

${ }^{2}$ Non-degenerate scoring rules are rules that never assign same score to different positions in a ranking, therefore these rules do not include plurality, k-approval rule etc.

${ }^{3}$ We omit the parenthesis whenever it is clear and write $R, R^{\prime}$ instead.
} 
maximal total scores:

$$
\alpha_{s}(p)=\max _{a \in A} \sum_{i \in N} \operatorname{score}(a, p(i))
$$

Example 1. Let $s=(m-1, m-2, \ldots, 0)$, then the Borda rule on each preference profile is defined as:

$$
\left.\alpha_{\text {Borda }}(p)=\arg \max _{a \in A} \sum_{i \in N} \operatorname{score}(a, p(i))=\arg \max _{a \in A} \sum_{i \in N}(m-a(i))\right)
$$

Let us now dwell upon the concepts of "closeness" between individual preferences and thereafter preference profiles. Let a function $\delta: \mathcal{L} \times \mathcal{L} \rightarrow \mathbb{R}$ assign a real number to each pair of preferences. A function over preferences is a distance function if it satisfies:

(i) Non-negativity: $\delta\left(R, R^{\prime}\right) \geq 0$ for all $R, R^{\prime} \in \mathcal{L}$,

(ii) Identity of indiscernibles: $\delta\left(R, R^{\prime}\right)=0$ if and only if $R=R^{\prime}$ for all $R, R^{\prime} \in \mathcal{L}$,

(iii) Symmetry: $\delta\left(R, R^{\prime}\right)=\delta\left(R, R^{\prime}\right)$ for all $R, R^{\prime} \in \mathcal{L}$.

(iv) Triangular inequality: $\delta\left(R, R^{\prime \prime}\right) \leq \delta\left(R, R^{\prime}\right)+\delta\left(R^{\prime}, R^{\prime \prime}\right)$ for all $R, R^{\prime}, R^{\prime \prime} \in \mathcal{L}$.

Two well-known examples of distance function are the discrete distance, and the swap distance. The former assigns 0 if the two preferences are identical, and 1 otherwise. The latter, characterized by Kemeny (1959) and Can and Storcken (2013), counts the symmetric total number of disjoint ordered pairs in preferences, or simply the number of "swaps of adjacent alternatives" required to transform one preference into another 4

Elkind et al. (2009) also refer to functions that satisfy i, ii, and iv only. These functions, which lack the symmetry condition, are called pseudo-distance functions. These functions may produce non-symmetric values for distances between some pair of rankings, i.e., $\delta\left(R, R^{\prime}\right) \neq \delta\left(R^{\prime}, R\right)$.

For distance rationalizability we will refer to distance functions between preference profiles. Given a distance function $\delta$ over preferences, a straightforward extension of $\delta$ over preference profiles, say $p, p^{\prime} \in \mathcal{L}^{N}$ can be defined as a function $d: \mathcal{L}^{N} \times \mathcal{L}^{N} \rightarrow \mathbb{R}$ as follows:

$$
d\left(p, p^{\prime}\right)=\sum_{i \in N} \delta\left(p(i), p^{\prime}(i)\right)
$$

Note that it is a very straightforward and common extension of distances over individuals preferences to distances over preference profiles, e.g., see Baigent (1987). We abuse notation for the sake of simplicity by referring to $\delta$ instead of $d$ as long as it is clear.

\footnotetext{
${ }^{4}$ In the literature, the swap distance and the Kemeny distance is interchangeably used. Kemeny (1959) originally assumes the distance for elementary changes to be 2 , whereas in many works, for convenience, this is normalized to 1. This occurs especially when the domain of preferences is strict and there is no indifference.
} 


\subsection{Distance rationalizability}

We consider only the "unanimity" as a consensus class. The definitions below are adapted smoothly to our notation for simplicity. For a more general notation that would be applicable to many other consensus classes, we refer the reader to Elkind et al. $(2009,2012)$.

Definition 1. $(\mathbf{U}, \delta)$-score: The unanimity-score of an alternative $a$ in a preference profile $p$ under the distance function $\delta$; is the minimal distance between the profile $p$ and any profile $p^{a}$ where $a$ is unanimity winner. Formally:

$$
(U, \delta)-\operatorname{score}(a, p)=\min _{p^{a} \in \mathcal{L}^{N}} \delta\left(p, p^{a}\right)
$$

Roughly speaking, $(U, \delta)$ - score of an alternative in a profile tells us how costly it is -in terms of a distance function- to make this alternative the best alternative in each individual preference, i.e., the unanimity winner. Obviously there are many possible preference profiles, $p^{a}$, where the alternative $a$ is the unanimity winner. The aforementioned score assigns the total cost to convert the original profile to one of such profiles for which the total cost is minimal. Next we reproduce the definition of distance rationalizability. We adapt again from Elkind et al. (2012) to simplify our notation.

Definition 2. A collective choice rule $\alpha$, is distance-rationalizable via unanimity and a distance function $\delta$, or simply $(U, \delta)$-rationalizable, if for all profiles $p \in \mathcal{L}^{N}$, we have:

$$
\alpha(p)=\arg \min _{a \in A}(U, \delta)-\operatorname{score}(a, p)
$$

To state verbally, a rule is $(\mathrm{U}, \delta)$-rationalizable if each outcome the rule assigns to each profile is also an alternative which have the minimal $(\mathrm{U}, \delta)$-score for that profile, i.e., the least costly to make the unanimity winner with that distance function.

\subsection{Weighted distances}

Can (2012) introduced weighted distances as an extension of the Kemeny distance on strict rankings, which would allow for differential treatment of the position of elementary changes. For instance consider, $R=a b c, R^{\prime}=a c b$, and $\bar{R}=b a c$. The Kemeny distance between $R$ and $R^{\prime}$ is 1 as well as the Kemeny distance between $R$ and $\bar{R}$. However one might argue that the former two is less dissimilar than the latter two, i.e., $\delta_{\omega}\left(R, R^{\prime}\right)<\delta \omega(R, \bar{R})$, because a swap at the top of rankings may be more critical than a swap at the bottom of thereof.

A weighted distance assigns weights to positions of such swaps with a weight vector on all possible swaps, e.g., $\omega=\left(\omega_{1}, \omega_{2}, \ldots, \omega_{m-1}\right)$. For any two rankings, then, that require more than a single swap, one would find the summation of sequential swaps on a shortest $5^{5}$ path between the two rankings. Hence a path between the two rankings is decomposed into elementary changes, and each elementary change is assigned its corresponding weight according to the weight vector.

For a technical description of the weighted distances, we refer the reader to Can (2012). Note that in the case of distance rationalizability, the complication regarding multiple paths between

\footnotetext{
${ }^{5}$ An example of the two possible shortest paths between $R=a b c$ and $R^{\prime}=c b a$ would then be $\rho_{1}=[a b c, b a c, b c a, c b a]$ and $\rho_{2}=[a b c, a c b, c a b, c b a]$.
} 
rankings do not occur. Hence, it is sufficient to illustrate a weighted distance with an example below:

Example 2. Let $R=a b c d$, and $R^{\prime}=$ dabc. Consider the weight vector $\omega=(10,3,1)$ and $a$ weighted distance $\delta_{\omega}$. Then:

$$
\begin{gathered}
\delta\left(R, R^{\prime}\right)=\delta(a b \mathbf{c d}, a b \mathbf{d} \mathbf{c})+\delta(a \mathbf{b d} c, a \mathbf{d} \mathbf{b} c)+\delta(\mathbf{a d} b c, \mathbf{d a} b c) \\
\delta\left(R, R^{\prime}\right)=\omega_{3}+\omega_{2}+\omega_{1}=10+3+1=14 .
\end{gathered}
$$

\section{Results}

Nitzan (1981) proved that the plurality rule is $\left(U, \delta_{\text {discrete }}\right)$-rationalizable and that Borda's rule is $\left(U, \delta_{\text {Kemeny }}\right)$-rationalizable. In this paper we extend the latter result to all non-degenerat ${ }^{6}$ scoring rules. In the sequel, we shall not use the term "non-degenerate" anymore to avoid repetition as long as it is clear. We show that any scoring rule is $\left(U, \delta_{\omega}\right)$-rationalizable where $\delta_{\omega}$, introduced in Can (2012), with particular weights. The class of weighted distance functions are characterized by two conditions on top of the usual metric conditions, i.e., positional neutrality and decomposability (see Can (2012)).

The results, herein, extend the existing interconnectedness (of the Borda rule and the Kemeny distance) to those between "all scoring rules" and "weighted distances". Weighted distances are Kemeny-like metrics which assign weights on the position of the swaps required to convert one (strict) ranking to another. In that respect Kemeny distance is also a weighted distance where weights on all possible swaps regardless of their positions are identical.

Elkind et al. (2009) show that all scoring rules including, Plurality, Borda, Veto, and k-approval, are "close" to being distance- rationalizable. They show that -if one does not insist on the symmetry condition- all these rules can be distance-rationalizable with pseudo-distances. We show that if we only focus on scoring rules that are non-degenerate, then it is fairly easy to find regular distance functions, satisfying symmetry as well, under which these scoring rules can be distance-rationalized. Hence our work somewhat compensates for the symmetry condition while however restricting the attention to non-degenerate rules.

Let $\alpha_{s}$ be a scoring choice rule with the scoring vector $s=\left(s_{1}, s_{2}, \ldots, s_{m}\right)$. Then consider a weighted distance $\delta_{\omega}$ with the weight vector $\omega=\Delta s=\left(s_{1}-s_{2}, s_{2}-s_{3}, \ldots, s_{m-1}-s_{m}\right)$, i.e., the weight assigned to each swap is the difference between the scores of the relevant consecutive positions. In the following theorem we explain the connection with the class of weighted distance functions and the distance rationalizability of non-degenerate scoring rules.

Theorem 1. A scoring rule $\alpha_{s}$ is $\left(U, \delta_{\omega}\right)$-rationalizable if $\omega=\Delta s$ satisfies weak decomposability.

Proof. Let $\delta=\delta_{\omega}$ be a weighted distance function with a weight vector $\omega=\Delta s=\left(s_{i}-s_{i+1}\right)_{i=1}^{m-1}$. We want to show that $\alpha_{s}$ is $\left(U, \delta_{\omega}\right)$-rationalizable which means for all profiles $p \in \mathcal{L}^{n}$, and for all alternatives $a \in A$, we have $a \in \alpha_{s}(p)$ if and only if $\left(U, \delta_{\omega}\right)$-score of $a$ is minimal for all $a \in A$. Take any $p \in \mathcal{L}^{n}$ and any $a \in A$. Now for each $i \in N$, let $\bar{p}^{a}(i) \in \mathcal{L}^{a}$ be such that $\bar{p}^{a}(i)$ is identical to $p(i)$ except that alternative $a$ is taken to top while everything else remains the same. By triangular

\footnotetext{
${ }^{6}$ By non-degenerate scoring rule we mean a non-degenerate scoring vector wherein $s_{i}>s_{i+1}$ for all $i=1,2, \ldots, m$.
} 
inequality of $\delta_{\omega}$, note that $\bar{p}^{a}(i)=\arg \min _{p^{a} \in \mathcal{L}^{a}} \delta_{\omega}\left(p(i), p^{a}\right)$, i.e., $\bar{p}^{a}(i)$ is the closest to $p(i)$ among all other preferences which have $a$ at the top. This is simply because when construction $\bar{p}^{a}(i)$, we leave everything unchanged except bringing $a$ to the top. Hence, for the constructed preference profile $\bar{p}^{a} \in \mathcal{L}^{N}$, the alternative $a$ is the unanimity winner and furthermore $\bar{p}^{a}$ is the closest to the original profile $p$ among all other profiles $p^{a} \in \mathcal{L}^{N}$ where $a$ is the unanimity winner.

Then, $\left(U, \delta_{\omega}\right)-\operatorname{score}(a, p)$ is $\sum_{i=1}^{n} \delta\left(p(i), \bar{p}^{a}(i)\right)$. By definition of a weighted distance and construction of $\omega$, this equals to $\sum_{i=1}^{n} \sum_{t=1}^{a(i)-1} \omega_{t}=\sum_{i=1}^{n} \sum_{t=1}^{a(i)-1}\left(s_{t}-s_{t+1}\right)$, which 7 in turn equals to $\sum_{i=1}^{n}\left(s_{1}-s_{a(i)}\right)=n \times s_{1}-\sum_{i=1}^{n} s_{a(i)}$. Note that the score of $a$ in $\alpha_{s}$ is $\sum_{i=1}^{n} s_{a(i)}$. Obviously, $n \times s_{1}-\sum_{i=1}^{n} s_{a(i)}$ is minimal if and only if $\sum_{i=1}^{n} s_{a(i)}$ is maximal. Hence $\left(U, \delta_{\omega}\right)-\operatorname{score}(a, p)$ is minimal if and only if $a \in \alpha_{s}(p)$. This completes the proof as the choice of $p$ and $a$ is arbitrary.

\section{References}

Baigent, N. (1987). Preference proximity and anonymous social choice. The Quarterly Journal of Economics, 161-169.

Can, B. (2012). Weighted distances between preferences. METEOR Research Memoranda $\mathrm{RM} / 12 / 056$.

Can, B. and T. Storcken (2013). A re-characterization of the kemeny distance. METEOR Research Memoranda RM/13/009.

Elkind, E., P. Faliszewski, and A. Slinko (2009). On distance rationalizability of some voting rules. In Proceedings of the 12th Conference on Theoretical Aspects of Rationality and Knowledge, pp. 108-117. ACM.

Elkind, E., P. Faliszewski, and A. Slinko (2012). Rationalizations of condorcet-consistent rules via distances of hamming type. Social Choice and Welfare, 1-15.

Kemeny, J. (1959). Mathematics without numbers. Daedalus 88(4), 577-591.

Meskanen, T. and H. Nurmi (2008). Closeness counts in social choice. Power, Freedom, and Voting, 289.

Nitzan, S. (1981). Some measures of closeness to unanimity and their implications. Theory and Decision 13(2), 129-138.

\footnotetext{
${ }^{7}$ Note that if $a$ is already at the top of $p(i)$, then this formulation gives 0 . The equation $\sum_{i=1}^{n} \sum_{t=1}^{a(i)-1} \omega_{t}$ sums the weights (costs) of carrying alternative $a$ to the top in each individual preference.
} 